\title{
Alfonso Perez-Agote (dir.), Portraits du catholicisme. Une comparaison européenne \& Yvon Tranvouez (dir.), La décomposition des chrétientés occidentales 1950-2010
}

Rennes, Presses universitaires de Rennes, coll. "Sciences des religions ", 2012, 178 p. \& Brest, Centre de Recherche Bretonne et Celtique, Université de Bretagne occidentale-Brest, 2014, 388 p.

\section{Frédéric Gugelot}

\section{(2) OpenEdition}

\section{Journals}

Édition électronique

URL : http://journals.openedition.org/assr/27431

DOI : $10.4000 /$ assr. 27431

ISSN : $1777-5825$

Éditeur

Éditions de l'EHESS

Édition imprimée

Date de publication : 1 octobre 2015

Pagination : 348

ISBN : 978-2-7132-2515-4

ISSN : 0335-5985

Référence électronique

Frédéric Gugelot, «Alfonso Perez-Agote (dir.), Portraits du catholicisme. Une comparaison européenne \& Yvon Tranvouez (dir.), La décomposition des chrétientés occidentales 1950-2010 ", Archives de sciences sociales des religions [En ligne], 172 | octobre-décembre, mis en ligne le 08 juin 2016, consulté le 24 septembre 2020. URL : http://journals.openedition.org/assr/27431 ; DOI : https://doi.org/ $10.4000 /$ assr. 27431

Ce document a été généré automatiquement le 24 septembre 2020.

(c) Archives de sciences sociales des religions 


\section{Alfonso Perez-Agote (dir.), Portraits du catholicisme. Une comparaison européenne \& Yvon Tranvouez (dir.), La décomposition des chrétientés occidentales 1950-2010}

Rennes, Presses universitaires de Rennes, coll. "Sciences des religions », 2012, 178 p. \& Brest, Centre de Recherche Bretonne et Celtique, Université de Bretagne occidentale-Brest, 2014, 388 p.

Frédéric Gugelot

\section{RÉFÉRENCE}

Alfonso Perez-Agote (dir.), Portraits du catholicisme. Une comparaison européenne, Rennes, Presses universitaires de Rennes, coll. «Sciences des religions », 2012, 178 p. Yvon Tranvouez (dir.), La décomposition des chrétientés occidentales 1950-2010, Brest, Centre de Recherche Bretonne et Celtique, Université de Bretagne occidentale-Brest, 2014, $388 \mathrm{p}$.

Partageant un identique constat des évolutions du catholicisme en Europe occidentale depuis le milieu du $\mathrm{xx}^{\mathrm{e}}$ siècle, ces deux ouvrages tentent de cerner les évolutions du catholicisme contemporain, décelable en particulier dans la fin des chrétientés. La baisse de la pratique dominicale, celle des vocations (qui diminue mécaniquement la capacité de l'Église à peser sur la société), des indices de baptêmes, mariages et même parfois d'enterrements illustrent une réelle déprise. Celle-ci est plus frappante encore dans les régions de chrétienté où l'assiduité aux offices, la fidélité aux rites saisonniers, l'autorité reconnue d'un clergé nombreux et visible, la fréquentation des institutions confessionnelles au sein d'une civilisation paroissiale où jusque-là la religion populaire 
était en adéquation avec le monde rural maintenaient des taux élevés de pratique. Le processus est identique avec des intensités et des chronologies différentes, en Vendée, Suisse romande, Belgique, Canada français et Québec, Irlande, Italie du Nord et Pays Basque.

2 L'ouvrage dirigé par Yvon Tranvouez (T) dévoile en trois temps - acteurs, territoires et images -, l'effacement de la configuration sociale des bastions catholiques qui avaient jusque-là résisté à la sécularisation des sociétés occidentales. Le livre dirigé par Alfonso Perez-Agote (PA.) établit le bilan après ce profond changement au tournant du $\mathrm{xx}^{\mathrm{e}}$ et $\mathrm{xxI}^{\mathrm{e}}$ siècle sur une zone géographique plus large, pays par pays.

3 Une chronologie déjà bien établie se retrouve : 1965-1975-1986 apparaissent comme des paliers de baisse de la pratique, puis de l'appartenance. Après un premier seuil dans les années 1965-1975 marqué par la crise des élites, avec un éloignement des croyances et des pratiques chez ceux-là mêmes qui avaient fait en sorte de les mettre au goût du jour, un net effet différé des abandons se dessine autour des années 1990, caractérisé par le détachement du tout-venant dans l'indifférence générale. Si Vatican II a conduit de nombreux militants à revendiquer un changement d'ecclésiologie, sa dynamique est devenue pour certains incontrôlable, les désillusions surviennent. Le conformisme social a mué : là où il était naturel d'être catholique, il est devenu naturel de ne pas l'être (T., p. 14). « La crise des chrétientés relève aussi de la crise, plus large, du discours et de la transmission » (T., p. 386). Des clercs et des militants jouent un rôle essentiel dans l'épuration du sentiment religieux traditionnel et donc de l'appartenance catholique longtemps perçue comme composante caractéristique de ces territoires ( $\mathrm{T}$., p. 16). Ils fondaient leur espoir dans l'avènement d'un laïcat responsable formé dans les mouvements d'Action catholique, qui vivrait enfin un christianisme accordé au monde moderne. Une génération au sens de communauté historique de mémoire et d'expérience aspire à une "religion du choix volontaire » (T., p.47) qui mène à l'éloignement de l'Église catholique. Tous les textes insistent sur la longueur du processus dont les racines sont lointaines même quand les phénomènes paraissent brutaux. La forte adéquation entre religion populaire et ruralité, sociabilité et conservatisme politique prouve que ces régions étaient parvenues à élaborer un système d'autorité fiable entre l'évêque et le prêtre, le prêtre et le fidèle, le militant et le pratiquant. Ces déprises seraient-elles simplement un « processus de normalisation " (T., p. 212)?

4 Même si les religieuses et les jeunes filles nombreuses à Boquen après la suppression de la clôture en 1966 sont souvent réduites aux tâches du quotidien, l'émancipation des femmes, « agents secrets de la modernité » (E. Morin, T., p. 196) est un processus décisif dans cette mutation du catholicisme. Elle se concrétise dans la limitation des naissances. En Suisse romande, le chanoine Joseph Putallaz et Agnès Bochatay diffusent la méthode de la température auprès des femmes qui permet, sans remettre en cause les principes de morale conjugale énoncés par l'Église, de déculpabiliser la recherche d'épanouissement du couple.

5 Au milieu des années 1960, atténuer l'angle confessionnel apparaît comme la solution pour attirer et retenir les jeunes au sein des mouvements de jeunesse en Alsace alors que les conflits de générations entravent la transmission au sein de la JOC. Ce qui confirme une faillite des instances de socialisation et de formation chrétienne. Une libération de la parole cléricale, dans un lourd silence des évêques bretons (T., p. 157), favorise l'expression de débats intenses autour de l'engagement à gauche, de la 
question du travail et du mariage des prêtres. Ainsi le déclin du modèle classique de recrutement, de formation et de statut de la prêtrise, n'est pas sur le moment perçu comme une perte, mais comme «le prix à payer de la dépolitisation et de la tranquillité » (T., p. 153).

Pour ces chrétientés, le processus est double. La décatholicisation est aussi une dénationalisation. Ces régions de forte identité catholique mêlent l'identité locale ou nationale avec le catholicisme. Les processus d'individualisation et de pluralisation des croyances brisent les régimes de religiosité ethnoculturelle. Au Canada, «le fait français et catholique s'étiole » (T., p. 251).

7 Au-delà des modèles nationaux, des phénomènes communs sont perceptibles dans le catholicisme européen. Le double mouvement de fort recul de la pratique et de vieillissement des clercs et des fidèles est l'aboutissement des évolutions évoquées avec des rythmes et des intensités différentes selon les contrées.

8 Pour la Belgique, le catholicisme reste « un monde » bien que l'Église comme institution se soit marginalisée. La distanciation à l'égard du catholicisme institutionnel conduit à une sécularisation individuelle fortement générationnelle (PA., p. 25 et T., p. 232). Les rites de passage catholiques sont néanmoins un peu considérés comme des services publics auxquels tout le monde "a droit» et certaines formes de religiosité dite populaire (pèlerinages) continuent de connaître un certain succès (PA., p. 27). La diminution du nombre de prêtres et les changements d'attitudes et d'organisation territoriale contraignent à faire largement appel à la société civile. Le monde catholique contribue à l'État providence (PA., p. 48). La société civile chrétienne reste bien ancrée en particulier dans la Flandre (T., p. 235) grâce à une modernisation intérieure des institutions catholiques qui parviennent à renouveler leur offre de services aux populations (PA., p. 19 et T. p. 237).

Malgré l'homogénéisation catholique de l'Espagne, qui perdure pratiquement jusqu'au $\mathrm{xx}^{\mathrm{e}}$ siècle, l'Église connaît aussi un affaiblissement progressif au niveau des pratiques, des croyances et des positionnements éthiques (divorce, avortement, euthanasie, homosexualité, à l'exclusion du suicide). Elle conserve une grande place à l'école, est très présente dans les ONG. Cet éloignement progressif de la population n'implique pas la disparition du catholicisme. Encore un quart de la population s'y rattache et l'institution ecclésiale reste très unie (PA., p. 99).

10 Après avoir offert en France «la matrice sociétale et symbolique qui a assuré la pérennisation des valeurs et références religieuses ", la culture catholique connaît les effets de la "sécularisation de la sécularisation ». La croyance en Dieu baisse depuis 1981, mais reste majoritaire, c'est l'affiliation à une confession qui s'estompe. La V ${ }^{\mathrm{e}}$ République marque un temps d'apaisement de la relation entre l'Église et l'État et une réévaluation de l'utilité socioéthique du religieux. Les attitudes ont changé : le religieux raisonnable apparaît moins comme une menace pour la liberté humaine et la cohésion sociale que comme l'un de leurs nécessaires adjuvants. D'autant que la permanence d'un imaginaire catholique certes folklorisé constitue une des limites de l'exculturation (PA., p. 131). L'enseignement catholique résiste en infléchissant son projet pédagogique en mettant l'accent sur la qualité de l'encadrement et du modèle éducatif proposé bien davantage que sur sa dimension confessionnelle. Les évêques sont très attentifs aux questions de bioéthique même si un subtil décalage avec Rome s'exprime dans une plus grande souplesse sur le terrain. L'affaiblissement de la présence catholique dans le 
monde social et politique s'accompagne d'une prégnance, continuée quoique discrète, de valeurs et de pratiques (PA., p. 151). Le « feuilletage » militant reste important.

11 Ce catholicisme connaît une dispersion du mode de pensée et d'agir dans deux types de positionnement: ceux qui font de leur croyance le facteur prédictif de leurs choix d'existence et ceux pour lesquels la foi n'est qu'une dimension latérale de la vie, sans effet réel sur la détermination de ses grandes orientations. Certains nourrissent l'espérance d'une rechristianisation du monde. Ils s'opposent à la société contemporaine, synonyme de déliquescence de la famille, rejettent la manipulation du vivant. Le subjectivisme contemporain favorise l'oubli de Dieu. L'autre courant, plus âgé que celui de la "nouvelle évangélisation ", a pour dessein d'accompagner le monde. Peuple avant d'être une hiérarchie, l'Église doit se faire modeste dans l'énonciation de ses propositions de sens; sa "doctrine sociale » est une source, mais pas une vérité indépassable surtout dans le domaine de l'intime - contraception, avortement, procréation assistée (PA., p. 157).

12 L'Italie illustre aussi la lente transformation d'une société à dominante catholique en société multireligieuse. L'appartenance au catholicisme de la plupart des Italiens (85\% de la population) résiste à l'épreuve du temps et de la modernité avancée mais un double pluralisme s'installe, pluralisme religieux et pluralisme dans les différentes formes de l'identité catholique italienne (PA., p. 165). La pratique religieuse est devenue minoritaire même si à un niveau élevé par rapport aux autres pays européens. La baisse des croyances est là aussi moins importante que celle des pratiques religieuses et du détachement de l'enseignement moral de l'Église en particulier en matière d'éthique sexuelle et familiale. Un fort besoin d'une foi sensible et visible, visionnaire et thaumaturge, s'exprime à la marge de l'Église officielle. Des déséquilibres dans la répartition géographique, un personnel plus féminin que masculin (une Église de sœurs plus que de prêtres), plus vieux que jeune, favorisent une façon plus autonome de vivre son appartenance religieuse, même si pour une majorité d'Italiens, il s'agit de concilier fidélité culturelle au catholicisme, autonomie dans la croyance et pluralité des formes d'appartenance à la religion-Église (PA., p. 202). Il s'agit donc moins d'une brutale rupture que d'une modalité différente pour retrouver du sens à l'action religieuse. La pluralité se dévoile aussi dans celle des militances catholiques. En Italie, la disparition du catholicisme politique avec la fin de la Démocratie chrétienne est partiellement compensée par l'explosion des mouvements de bénévolat. Plus récemment, un engagement de type culturel et identitaire autour des thèmes éthiques de la tradition catholique (famille, bioéthique, limites de la science, éducation) domine.

Parmi les militants, deux sensibilités se détachent sur la façon dont la foi et l'Église doivent s'insérer dans la modernité avancée. La première dénonce fortement le sécularisme et le relativisme éthique, résolue à s'opposer à un monde qui tend à effacer Dieu de l'espace public (PA., p. 204). La seconde revendique une foi dans le monde plus vécue qu'affirmée. L'espérance d'une renaissance religieuse éloigne les premiers de l'engagement social et politique des seconds, car leur charisme les pousse à se concentrer sur l'eschatologie chrétienne, la recherche radicale de la sainteté, la conversion personnelle plutôt que sur l'action dans le monde.

14 Au Portugal, le processus est proche. L'identité nationale reste profondément liée à l'univers culturel catholique malgré le déclin du recours aux rites de passage (PA., p. 214). L'Église continue d'exercer un rôle social essentiel et reste très compétitive 
parmi les institutions productrices de sens, d'autant qu'elle apparait plus diverse et plurielle avec les nouveaux mouvements ecclésiaux (Focolari, charismatiques).

Des traits se retrouvent pour tous les pays du panel : une perte de substance avec une réelle baisse de la pratique et du nombre des fidèles, une institution moins nombreuse et donc plus lointaine (encadrement réduit, hiérarchie moins audible) face à une société libérale avancée (divorce, avortement). Le catholicisme maintient néanmoins des structures, en particulier d'interventions sociales et culturelles (presse, éducation) accordant une place plus grande à une pluralisation plus prononcée au sein de l'Église. Les valeurs chrétiennes conservent une puissance d'imprégnation culturelle et participent d'un "vivre-ensemble» durable dans une société désormais plurielle. L'Église préserve une légitimité dans l'espace public en tant qu'autorité morale (T., p. 299). Une nouvelle figure de chrétienté réduite à des groupes d'élection moins ancrés dans un territoire revendique une culture et une identité marquées du sceau catholique. Ils mobilisent des ressources symboliques locales, mais de façon patrimoniale dans une optique de réinvention identitaire (les scouts Bleimor) ou de réaffirmation religieuse (T., p. 388) tandis que la Ligue du Nord en Italie soutient une religion territoriale qui présente parfois des aspects panthéistes (T., p. 285).

Ces deux ouvrages qui se complètent heureusement permettent de cerner les traits de l'évolution du catholicisme sur ce large demi-siècle où s'estompe l'Église du xix siècle pour dessiner une autre modalité d'être catholique en Europe occidentale, plus diverse. Les processus dégagés dans l'ouvrage de Tranvouez se retrouvent dans celui de PerezAgote. Les croyances et les pratiques procèdent aujourd'hui de plus en plus des seules exégèses individuelles et s'éprouvent dans des communautés, ou dans des réseaux, souvent fluides, plastiques, qui peinent à rassembler, malgré les desseins unificateurs de la hiérarchie, dans un espace commun d'actions et de paroles.

17 Ces livres permettent de dessiner la pluralité interne du catholicisme et les modes d'organisation de l'institution pour à la fois encadrer et faire vivre cette complexité. Elle est la preuve de ce régime de tension interne au catholicisme, qui tout à la fois le déchire et le fait vivre. 